Bio - grafía. Escritos sobre la Biología y su Enseñanza. ISSN 2027-1034

Edición Extraordinaria. p.p. 92 - 99

Memorias del IX Encuentro Nacional de Experiencias en Enseñanza de la Biología y la

Educación Ambiental. IV Congreso Nacional de Investigación en Enseñanza de la Biología.

\title{
LAENSEÑANZA DE LO VIVO Y LA VIDA DESDE LAS COLECCIONES BIOLÓGICAS: UNA OPORTUNIDAD PARA PENSAR LAS COLECCIONES DESDE LA COMPLEJIDAD
}

\section{TEACHING ABOUT LIFE, AND LIFE FROM BIOLOGICAL'S COLLECTION: AN OPPORTUNITY FOR THINKING THE COLLECTION FROM COMPLETION}

\author{
Mendoza, Elkin \& Velandia, Andrea ${ }^{1}$
}

\section{RESUMEN}

En el presente artículo investigativo se muestran los resultados parciales del proyecto de investigación: "Estrategia pedagógica para la enseñanza de la vida y lo vivo a partir de las colecciones biológicas: Hacia la configuración del Museo Pedagógico de Biología", en el que se han desarrollado categorías conceptuales como son las colecciones biológicas y la enseñanza de lo vivo y la vida; a partir de las cuales se hace un primer análisis de las principales tendencias en los trabajos de grado desarrollados en el departamento de biología de la Universidad Pedagógica Nacional que hasta el momento se han revisado desde elementos de la caja de herramienta arqueológica-genealógica, permitiendo de esta manera plantear cuál podría ser la perspectiva de las colecciones biológicas a propósito de la enseñanza de la vida y lo vivo.

PALABRAS CLAVE: Enseñanza de lo vivo y la vida, colecciones biológicas, museo.

\section{ABSTRACT}

In the follow, investigative essay shows the results for investigative projects. "A pedagogical strategy for teaching about life, and life from biological's collection: to the Teaching Museum of Biology ". It has been developed conception of categories for biological's collection and teaching about what live and life. On base of this, a first analysis is made from looking the senior thesis' tendencies at the University Pedagogica Nacional. So far, it has been investigated about element like a tools box for archeology and genealogy, so it shows a possible perspective of the biological's collection focusing on the area of teaching about what live and life.

KEY WORDS: Teaching about what live and life, biological's collection and museum

\footnotetext{
${ }^{1}$ Licenciados en biología. Monitores de investigación. Título del proyecto: Estrategia pedagógica para la enseñanza de la vida y lo vivo a partir de las colecciones biológicas: Hacia la configuración del Museo Pedagógico de Biología. Grupos de investigación: CASCADA, Grupo Estudios en Enseñanza de la Biol ogía.
} 


\section{Bio - grafía. Escritos sobre la Biología y su Enseñanza. ISSN 2027-1034}

Edición Extraordinaria. p.p. 92 - 99

Memorias del IX Encuentro Nacional de Experiencias en Enseñanza de la Biología y la

Educación Ambiental. IV Congreso Nacional de Investigación en Enseñanza de la Biología.

\section{INTRODUCCIÓN}

El presente artículo investigativo surge desde la experiencia que como monitores de investigación se ha tenido hasta el momento y que posibilita abrirse a perspectivas diferentes en cuanto a nociones y conceptos abordados en la enseñanza de la biología, a partir de procesos de reflexión que conllevan a pensar las colecciones desde la memoria y la historia, posibilitando movilizar los saberes acerca de la vida y lo vivo.

Para esto se han desarrollado las categorías conceptuales enseñanza de lo vivo y la vida y de las colecciones biológicas, permitiendo así configurar una noción de éstas que vaya acorde con el desarrollo del proyecto investigativo "Estrategia pedagógica para la enseñanza de la vida y lo vivo a partir de las colecciones biológicas: Hacia la configuración del Museo Pedagógico de Biología". Además, se ha ido desarrollando la tematización de los trabajos de grado con el fin de identificar los elementos pedagógicos y didácticos que los estudiantes desarrollan a partir de colecciones y museos, así como también de las principales tendencias.

Finalmente, se plantea que es posible transformar la manera de entender las colecciones biológicas al articularlas desde la enseñanza de lo vivo y la vida y no exclusivamente enfocado hacia la investigación de tipo biológico. Proceso que a su vez posibilita una noción de museo diferente que se encuentre atravesado por aspectos como la historia y la memoria, lo cual conlleva a pensar el museo dinámicamente y como escenario de reflexión.

\section{METODOLOGÍA}

El presente trabajo de investigación se ha desarrollado desde un enfoque cualitativo en el que se toman elementos propuestos en la caja de herramientas de la mirada arqueológica genealógica y a partir de los cuales se realiza la revisión de los trabajos de grado, permitiendo identificar las principales tendencias con relación al trabajo con museos y colecciones biológicas.

Los elementos que hasta el momento se han tenido en cuenta para el desarrollo del proyecto de investigación "Estrategia pedagógica para la enseñanza de la vida y lo vivo a partir de las colecciones biológicas: Hacia la configuración del Museo Pedagógico de Biología" son la tematización y elaboración de matrices juntos con la construcción de categorías e identificación de elementos pedagógicos y didácticos.

Con la tematización y elaboración de matrices se busca desarticular los textos en sus temáticas centrales posibilitando de esta manera, cruces horizontales entre los documentos y la estructuración de agrupamientos temáticos; además, de los diversos fragmentos de los documentos, se señalaron las acciones de poder, sujetos e instituciones, que permitirán da0r cuenta en la continuidad del proyecto de diferentes hechos y acontecimientos así como de una matriz correspondiente a poder y subjetividad (Castro, 2007).

Otro de los elementos trabajados en el desarrollo del proyecto es la construcción de categorías de la investigación y de su respectiva orientación, permitiendo consolidar las 


\title{
Bio - grafía. Escritos sobre la Biología y su Enseñanza. ISSN 2027-1034
}

Edición Extraordinaria. p.p. 92 - 99

\author{
Memorias del IX Encuentro Nacional de Experiencias en Enseñanza de la Biología y la \\ Educación Ambiental. IV Congreso Nacional de Investigación en Enseñanza de la \\ Biología.
}

diferentes perspectivas que hay en torno a éstas y a su vez delimitar en qué sentido se desean asumir, permitiendo la configuración del trabajo investigativo.

\section{RESULTADOS}

En la enseñanza de la biología se destacan dos nociones: la enseñanza de la vida y la enseñanza de lo vivo, que si bien pueden resaltarse como dos aspectos diferentes desde fundamentos filosóficos y epistemológicos, es posible ver que en la práctica de la enseñanza de la biología se abordan desde su relación en tanto lo vivo hace parte de la vida, más aún cuando se contempla que el proceso de enseñanza involucra el contexto, trascendiendo los límites naturales para abordar aquellos en los que está también la cultura, aspectos sociales y en definitiva todo lo que influye en la formación de las personas.

En este sentido, teniendo en cuenta la postura desde la cual se orienta el desarrollo del proyecto de investigación y en conformidad con lo encontrado en la revisión de documentos y trabajos de grado del Departamento de Biología acerca de la Enseñanza de la Biología, es necesario mostrar cómo se entiende lo vivo y la vida. Cuando se hace referencia a la enseñanza de lo vivo hay un énfasis en lo particular del conocimiento disciplinar, en donde la configuración del concepto de lo vivo tiene lugar cuando se integran los órganos con las funciones, posibilitando así la existencia del organismo. Además, esta noción de vivo se ve apoyada cuando los naturalistas se centraban en observar, describir y clasificar los seres vivos.

Por otra parte, cuando se habla de la enseñanza de la Biología desde la noción de que surgen y desarrolla el ser humano en su relación con la naturaleza, por lo que se posibilita una comprensión de la biología más allá del contenido disciplinar. Es por esto que, en la enseñanza de la biología bajo un enfoque intercultural se permite reconocer otras formas de concebir la vida y lo vivo, otras nociones que se van configurando a partir de las prácticas en las comunidades.

Esta comprensión de la biología desde la enseñanza de la vida y lo vivo, en conformidad con Cárdenas (2014):

Se hace pertinente en la medida en que se parte del contexto y la realidad de quienes experimentan las situaciones y dinámicas que se desenvuelven en el diario vivir con la intención siempre de comprender los saberes y conocimientos locales y establecer su importancia dentro de los conocimientos occidentales y homogenizantes de la sociedad occidental, aproximándonos así a la caracterización de aspectos epistemológicos y ontológicos del conocimiento de la vida y de lo vivo en un país que se considera biodiverso, pluriétnico y multicultural. (p. 37)

Las características de un país como Colombia en cuanto a diversidad biológica y cultural abren la posibilidad para que los profesores de esta ciencia reconozcan lo vivo como su pilar fundamental, donde el conocimiento configura su quehacer profesional y da lugar a que integre en la práctica el contexto, aquellas relaciones sociales y culturales en las cuales se da la formación de la persona. (Vanegas \& Barrera, 2013). Por lo tanto, el 


\title{
Bio - grafía. Escritos sobre la Biología y su Enseñanza. ISSN 2027-1034
}

Edición Extraordinaria. p.p. 92 - 99

\author{
Memorias del IX Encuentro Nacional de Experiencias en Enseñanza de la Biología y la \\ Educación Ambiental. IV Congreso Nacional de Investigación en Enseñanza de la \\ Biología.
}

profesor es sujeto transversal en la enseñanza de la biología en la medida que permite desarrollar procesos en los que los niños integren saberes acerca de lo vivo y la vida, logrando además el planteamiento de propuestas pedagógicas y didácticas. Esto se evidencia cuando

El trabajo desarrollado con los niños conduce a reflexionar que los maestros de biología no solo se deben enfocar en los procesos biológicos, sino también deben tener en cuenta las relaciones de lo vivo con el entorno, (...) esto indica la importancia de tener en cuenta estos aspectos en la enseñanza de la biología, pues las concepciones que los estudiantes tienen acerca de este tema se convierten en un pilar que debe orientar los procesos didácticos en la enseñanza de esta ciencia. (Jiménez, 2013, p. 74)

A partir de esto, se articula en los procesos de enseñanza de la vida y lo vivo distintas maneras de abordar esta ciencia, tal es el caso de la paleobiología y el bioarte que permiten tanto expresar como dar a conocer el objeto de estudio de la biología, de manera que se ocurra un proceso de comprensión de la naturaleza en conformidad con las dinámicas culturales y sociales del ser humano. En conformidad con Becerra (2013), desde la paleobiología se

contempla el estudio y comprensión de la evolución desde el uso de los fósiles, como recursos, que suelen despertar su interés y les permite, tener evidencia de parte de la historia de la vida, de cómo eran los organismos y los ambientes en el pasado, entre otros, lo cual pueden relacionar con otros temas y conceptos que forman parte tanto de su realidad como de la biología. (p. 23)

Por otra parte, está la configuración del arte en relación con la biología, en dónde Jaramillo, C. (2012) menciona que esta se encuentra en "la indiscutible belleza de lo vivo desde sus formas, colores, expresiones y relaciones." (p. 246.) Pensando así en la posibilidad de una relación aún más amplia desde la estética, los colores y que abre camino a expresar de distinta manera la vida y lo vivo. Con esto, se consolida el bioarte en donde se desarrollan un conjunto de prácticas artísticas como forma para mostrar la naturaleza y sus dinámicas, lo cual trae consigo la interdisciplinariedad y no sólo con relación al arte, también puede ser con otras ciencias como la filosofía, la matemática y la física en donde se pone en común todos los medios y conceptos que surgen de relacionarse entre ciencias para la comprensión del mundo y de la vida. (Benítez, 2013).

Dentro de las formas de abordar la enseñanza de la vida y lo vivo se encuentran las colecciones biológicas, desde las que surgen distintas apuestas didácticas en las que se pueden identificar elementos pedagógicos que buscan cambiar la actitud de los estudiantes hacia el conocimiento disciplinar y así establecer otras relaciones con lo vivo desde su cotidianidad, para finalmente reconocer la diversidad biológica. Sin embargo, son diversas las perspectivas que se pueden distinguir en las colecciones biológicas, dentro de las cuales se puede mencionar: como bancos de información y de datos, patrimonio nacional, reconocimiento de la biodiversidad, conjunto de especímenes y ejemplares, como lugar y opción de conocimiento y finalmente como una opción de aprendizaje.

Esta última perspectiva es la que más interesa en el desarrollo del proyecto investigativo, 


\title{
Bio - grafía. Escritos sobre la Biología y su Enseñanza. ISSN 2027-1034
}

Edición Extraordinaria. p.p. 92 - 99

\author{
Memorias del IX Encuentro Nacional de Experiencias en Enseñanza de la Biología y la \\ Educación Ambiental. IV Congreso Nacional de Investigación en Enseñanza de la \\ Biología.
}

puesto que se plantea desde la enseñanza de la biología y así mismo en dirección a configurar el museo pedagógico de esta ciencia, la posibilidad de construir memorias con relación a las colecciones, una memoria que no sólo involucra el pasado, sino que hace partícipe el presente y los diversos acontecimientos que una persona puede integrar al momento de observar las colecciones biológicas, por lo que se permite considerar otras formas de conocimiento que se desarrollan en universidades y centro de formación.

De esta manera, las colecciones se toman en la educación como herramientas y material de apoyo en actividades que posibilitan a los estudiantes reconocer la Entonces, las colecciones biológicas asumidas desde la enseñanza de la biología posibilitan el conocimiento de la biodiversidad, así como el planteamiento y desarrollo de estrategias, propuestas, actividades que tienen como finalidad contribuir a la educación y formación de las personas, pues no sólo los estudiantes pueden hacer parte de este proceso, sino la sociedad en general. Por lo tanto, las colecciones biológicas no sólo se reducen al conocimiento de tipo científico sino a otras formas de saber en las que es partícipe la sociedad en general y que pueden contribuir a las finalidades del conocimiento de la diversidad biológica como es su cuidado y conservación, lo cual trae consigo el cuidado de la naturaleza.

En este sentido, las colecciones dejan de entenderse desde lo meramente disciplinario cuando se abordan a partir de la enseñabilidad de lo vivo y de la vida, lo cual lleva a cuestionarse por elementos pedagógicos y didácticos que se pueden identificar en las prácticas de los licenciados en formación así como del material que producen en su quehacer, ejemplo de esto son prototipos, colecciones de cantos, manuales ilustrados, biogeografía cultural, etc, los cuales propenden por el respeto de la vida, visibilizando así apropiaciones sociales de la biología que superan las tradiciones científicas y recogen los saberes locales y contextuales.

Como evidencia de la manera en que se han entendido las colecciones biológicas se encuentran los trabajos de grado desarrollados en el departamento de Biología de la Universidad Pedagógica Nacional, a partir de los cuales es posible identificar que, en términos de organización de las colecciones, estas se clasifican de acuerdo con los siguientes criterios: seres vivos y no vivos, la clase a la que pertenecen y por las relaciones jerárquicas y evolutivas, promoviendo a partir de la organización de los ejemplares de manera coherente, rigurosa y explicativa, una perspectiva de museo como un todo. Dicha noción se fomenta mediante el desarrollo de actividades como talleres, visitas guiadas, salidas de campo y reseñas científicas, de tal manera que el educando tenga una visión general del museo, pueda conocer su estructura y su importancia, así como también, desarrolle actitudes para el conocimiento y conservación de la fauna y flora del país.

Un rasgo distintivo de algunos de los trabajos de grado desarrollados en los años 90, en relación con el museo, es que se plantean para la enseñanza en ciencias naturales mas no de biología, aspecto que puede tenerse en cuenta hoy en día cuando una vez más ocurre lo mismo y que indiscutiblemente invita a cuestionarse por el objeto de estudio de este tipo de enseñanza.

Entonces, tanto el museo y específicamente las colecciones como son los herbarios, se 


\section{Bio - grafía. Escritos sobre la Biología y su Enseñanza. ISSN 2027-1034}

Edición Extraordinaria. p.p. 92 - 99

Memorias del IX Encuentro Nacional de Experiencias en Enseñanza de la Biología y la

Educación Ambiental. IV Congreso Nacional de Investigación en Enseñanza de la Biología.

consideran en este tiempo como alternativas pedagógicas para la enseñanza de las Ciencias Naturales, que pueden integrarse al programa curricular con el fin de despertar el interés y la curiosidad del visitante así como de su principal función educativa "contribuir a la formación cultural, intelectual y afectiva del público en general, desarrollando en éste una nueva sensibilidad tanto hacia el mundo que lo rodea como de conocer su pasado" (Espitia, 1990, p. 21.)

Desde los museos y colecciones surge material educativo como son las guías ilustradas, talleres, reseñas científicas que se mencionaron anteriormente, pero mientras que estas últimas se desarrollan con la finalidad de entender el museo como un todo a partir de la organización teórica, práctica, coherente, rigurosa y explicativa, las guías ilustradas se establecen como material que permite fortalecer la enseñanza de los conceptos de sistemática y taxonomía en el marco de la enseñanza de las ciencias naturales, a la vez que permite valorar las colecciones y propiciar actitudes que contribuyan a su conocimiento y conservación.

Es necesario destacar que las guías ilustradas se conciben como parte de una estrategia educativa con el fin de rescatar el museo de biología y de fortalecer habilidades científicas como la observación, la curiosidad y manipulación de las colecciones pero que no se queda en la apropiación de las ciencias naturales, sino que allí también se entretejen relaciones interpersonales, por to que el museo adquiere un rol especial como es informador, comunicador, motivador y recreador.

\section{DISCUSIÓN}

A partir de la revisión de los trabajos de grado se puede reconocer que desde las colecciones biológicas se plantean recursos didácticos como guías ilustradas, cartillas, unidades y talleres; así como la tendencia a organizar y determinar los ejemplares de las diversas colecciones biológicas. En este sentido, la principal orientación al abordar las colecciones es hacia la investigación de tipo biológico, por lo que es posible pensar que las prácticas y trabajos desarrollados por los estudiantes se reducen al aprendizaje exclusivamente de lo vivo, dejando de lado el contexto de los sujetos y las diversas relaciones que influyen en el conocimiento biológico.

Surge de esta manera, el propiciar una visión de las colecciones biológicas no sólo estudiantes en los que se encuentran comprometidas sus formas de ser y actuar, de tal forma que, el profesor desarrolla su quehacer teniendo presente las diversas dinámicas en las que se desenvuelven los estudiantes y la sociedad en general. Con esto, el profesor puede hacer de la biología, a través de apuestas didácticas y pedagógicas, una oportunidad para establecer relaciones con lo vivo desde la cotidianidad de los sujetos e incluso un cambio de actitud hacia el conocimiento pedagógico disciplinar.

Por lo tanto, la reflexión y estudio de lo vivo en la biología también se puede comprender desde la configuración de vida de los sujetos, puesto que son diversos los saberes y prácticas culturales que surgen en la conformación de la sociedad humana que tiene repercusión en la naturaleza, pero así mismo en el ser humano, en otras palabras, tiene influencia en la otredad. Materialización de estos procesos de reflexión y estudio pueden 


\title{
Bio - grafía. Escritos sobre la Biología y su Enseñanza. ISSN 2027-1034
}

Edición Extraordinaria. p.p. 92 - 99

\author{
Memorias del IX Encuentro Nacional de Experiencias en Enseñanza de la Biología y la \\ Educación Ambiental. IV Congreso Nacional de Investigación en Enseñanza de la \\ Biología.
}

ser las colecciones biológicas, en tanto pueden ser la oportunidad para que desde allí se posibiliten experiencias en las que se reconozcan estas prácticas y se integre lo vivo con la vida.

Lo anterior será posible si se pasa de una perspectiva reducida de la vida y lo vivo a una que se caracterice por la complejidad, en la que se reconozcan las diversas relaciones y se asuma la formación de los licenciados en biología no sólo desde el conocimiento disciplinar sino como resultado de saberes relacionales que atraviesan las prácticas de vida de los sujetos.

Esto no quiere decir que en la formación de los futuros profesores no se estén desarrollando elementos pedagógicos y didácticos, sino que a partir de lo identificado en la revisión de los trabajos de grado y en la configuración de las categorías conceptuales del trabajo investigativo, hay una tendencia a orientarlos hacia lo que podría denominarse lo vivo, dejando así de lado la vida.

Finalmente, cabe mencionar que si es posible transformar la perspectiva que se tiene y ha predominado de las colecciones así como de la orientación de los trabajos realizados en torno a las mismas, seguramente la mirada que se tiene de los museos también se transforme, logrando que no se vean únicamente como lugares en los que se conservan objetos, especímenes o ejemplares, o desde los cuales se desarrollan investigaciones para ampliar la información que se encuentra allí, sino también como escenario de reflexión y configuración de la persona, en el que la historia, la memoria y la experiencia son fundamentales en tanto permiten a la persona integrar lo que observa con la complejidad de su vida.

\section{CONCLUSIONES}

Entender la vida y lo vivo permite configurar otras dinámicas que giran en torno a las colecciones, en dónde la vida, permite entrelazar la memoria y la historia que conforma diversos saberes, los cuales van sujetos a los recuerdos y sentidos que configuran el museo; permitiendo desde lo vivo no centrarse únicamente desde el organismo y pensarlo a nivel biológico, sino que propicia encuentros entre lo que se halla en el museo y la configuración de los sujetos en este.

En esta medida, las colecciones permiten relacionar la mirada polisémica de la vida y lo vivo, tejiendo su esencia desde el presente evocando al pasado, en donde los sujetos reconstruyan las diversas prácticas que han girado en torno a la colección y al museo, permitiendo dialogar con los sujetos desde diversas miradas; hablando propiamente dicho, que aquella colección evocando lo vivo desde el organismo, el aprendizaje, la biología en sí, y la vida misma, no solo desde aquellas prácticas de la enseñanza, si no desde las reflexiones de los sujetos en torno a esta; dando paso a si, a un museo que hable por sí solo y a una colección que evoque pensarse desde diferentes lugares.

Es necesario considerar que la enseñanza de lo vivo y la vida desde las colecciones puede propiciar diferentes posibilidades para pensar la formación de los maestros en donde se integran elementos pedagógicos y didácticos en conformidad con la 
Bio - grafía. Escritos sobre la Biología y su Enseñanza. ISSN 2027-1034

Edición Extraordinaria. p.p. 92 - 99

Memorias del IX Encuentro Nacional de Experiencias en Enseñanza de la Biología y la

Educación Ambiental. IV Congreso Nacional de Investigación en Enseñanza de la Biología.

contemporaneidad.

Finalmente, el proceso investigativo, conlleva a tejer diversas prácticas que posibilitan seguir pensando las colecciones en pro de entender la vida y lo vivo desde una mirada polisémica en la que las colecciones biológicas se hallan

\section{BIBLIOGRAFÍA}

- Becerra, J. (2013) Enseñanza de la biología - lo vivo y la vida- origen y evolución. (Tesis de pregrado). Universidad Pedagógica Nacional, Chorrera, Colombia.

- Benítez, L (2013). Bioarte. Una estética de la desorganización. Tesis Doctoral. Universidad Autónoma de Barcelona, España.

- Cárdenas, A. (2014). Aprendiendo Y Sembrando Para La Vida -lachaikuspa Tarpuspa Kausaimanda- Como espacios de aprendizaje de la vida y de lo vivo desde un enfoque intercultural. (Tesis de pregrado). Universidad Pedagógica Nacional, Bogotá, Colombia.

- Jaramillo, C. (2012). Formas y fuerzas de la experiencia estética: pinceladas, matices y posibilidades en la formación de maestros de Biología de la Universidad Pedagógica Nacional. (Tesis de pregrado). Universidad Pedagógica Nacional, Bogotá, Colombia.

- Jiménez, L. (2013). Concepciones acerca de lo vivo en dos contextos culturalmente diversos, como aporte a la reflexión de la enseñanza de la biología en Colombia. (Tesis de pregrado). Universidad Pedagógica Nacional, Bogotá, Colombia.

- Orjuela, L \& Rivera, Y. (2009). Ideas acerca de lo vivo en los estudiantes de grado primero y segundo de primaria de la escuela rural IED Verjón alto (choachí) y escuela urbana IED Rafael Núñez (Bogotá). (Tesis de pregrado). Universidad Pedagógica Nacional, Bogotá, Colombia.

- Puentes, K. (2011). Implementación Y Validación De Una Propuesta Didáctica Para La Comprensión Del Concepto Sistema Vivo Con Estudiantes De Grado Sexto (Ciclo Tres) Del Liceo Maranata. (Tesis de pregrado). Universidad Pedagógica Nacional, Bogotá, Colombia.

- Rincón, L. (2013). Concepciones acerca de lo vivo en dos contextos culturalmente diversos, como aporte a la reflexión de la enseñanza de la biología en Colombia. (Tesis de pregrado). Universidad Pedagógica Nacional, Bogotá, Colombia.

- Serrato, D. (2012). La botánica como saber escolar: condiciones de posibilidad relacionadas con su desplazamiento del currículo escolar colombiano entre 1955 y 1965. (Tesis de pregrado). Universidad Pedagógica Nacional, Bogotá, Colombia.

- Venegas, A \& Barrera, I. (2013). Están vivos porque tienen vida: lo que nos dicen las concepciones de los niños y niñas del aula de aceleración del Colegio Diego Montaña Cuéllar I.E.D. (Tesis de pregrado). Universidad Pedagógica Nacional. Licenciatura En Biología. Bogotá. 